\title{
DSP Embedded Smart Surveillance Sensor with Robust SWAD-based Tracker
}

\author{
Gaetano Di Caterina ${ }^{1}$, Iain Hunter ${ }^{2}$, and John J. Soraghan ${ }^{1}$ \\ 1 Department of Electronic and Electrical Engineering, University of Strathclyde, \\ 204 George Street, Glasgow, G1 1XW, UK \\ 2 Texas Instruments Limited, 800 Pavilion Drive, Northampton, NN4 7YL, UK
}

\begin{abstract}
Smart video analytics algorithms can be embedded within surveillance sensors for fast in-camera processing. This paper presents a DSP embedded video analytics system for object and people tracking, using a PTZ camera. The tracking algorithm is based on adaptive template matching and it employs a novel Sum of Weighted Absolute Differences. The video analytics is implemented on the DSP board DM6437 EVM and it automatically controls the PTZ camera, to keep the target central to the field of view. The EVM is connected to the network and the tracking algorithm can be remotely activated, so that the PTZ enhanced with the DSP embedded video analytics becomes a smart surveillance sensor. The system runs in real-time and simulation results demonstrate that the described SWAD outperforms other template matching measures in terms of efficiency and accuracy.
\end{abstract}

\section{Introduction}

Modern digital video surveillance has made possible the semantic analysis of video data via software, on computer systems, using Video Analytics [1,2]. As digital circuits become smaller and faster, video surveillance sensor systems can be equipped with processing functionalities and DSPs, so that smart video analytics algorithms can be embedded within the surveillance sensors, for fast incamera processing. Such sensors are referred to as smart cameras and they can exchange information with each other, to form a collaborative network of smart surveillance sensors, where the human intervention is reduced to the minimum.

Starting from the work described in [3], in this paper we present a DSP embedded implementation of an adaptive template matching algorithm for object and people tracking, in the context of a smart surveillance sensor including a pan-tilt-zoom (PTZ) camera. The novel Sum of Weighted Absolute Differences (SWAD) proposed in [4] for robust target tracking is implemented in real-time on the single core DSP board DM6437 Evaluation Module (EVM) from Spectrum Digital. When compared to other tracking methods, such as Sum of Absolute Difference (SAD), Normalized Cross-Correlation (NCC) and Mean Shift tracker (MS), the SWAD-based algorithm shows better performance in the context of an embedded implementation, as it exploits the DM6437 fixed-point architecture. 
The video analytics algorithm on the EVM automatically controls the PTZ camera to follow the target and keep it central to the field of view, when it moves towards the frame boundaries. The EVM is connected to the network and, differently from the system in [3], the tracker can be activated and deactivated from remote, through on/off TCP-based messages. The contribution of this paper is therefore threefold: first, a real-time DSP embedded implementation of the SWAD-based tracker described in [4] is presented; second, extended performance evaluation of the SWAD-based tracker compared with SAD, NCC and MS is provided; third, the smart surveillance system in [3] is improved with remote activation, faster processing and better tracking performance, to create, in conjunction with the PTZ camera, an embedded smart surveillance sensor.

The remainder of the paper is organized as follows. Section 2 reviews related work in the field of tracking algorithms and embedded surveillance applications. An overview of the DSP embedded system described in this paper is given in section 3, while section 4 describes the video analytics implemented on the DSP, including tracking algorithm and camera controller. Section 5 provides a quantitative performance evaluation of the system, while section 6 concludes the paper.

\section{Related work}

The implementation of image processing algorithms on embedded platforms poses challenges due to hardware limitations and time constraints. However, a number of implementation techniques were introduced to overcome these issues [5-7]. Previous work using static cameras [8-10] describe the implementation of smart surveillance sensors, for example for object detection and tracking.

The usage of active cameras for tracking applications is described in [11-14], but these mainly focus on the algorithm itself, rather than on creating an actual embedded smart sensor, as in the case of the work presented in this paper. To the best of the authors' knowledge, only the work in [15] has implemented a tracking algorithm [16] to control an active camera on an embedded device, namely an FPGA. However, performance evaluation and comparison with other algorithms or systems are missing from both [15] and [16]. Moreover, the work in [15] comprises an implementation exercise of a given tracking algorithm: the implementation choices on the FPGA are driven by the characteristics of the selected algorithm, rather than the algorithm itself being tailored for specific hardware requirements. In contrast, the video analytics system presented in this paper is embedded on a single core DSP board and such real-time implementation serves more as a proof of concept, i.e. prove that the described SWAD-based tracker achieves better performance compared with other algorithms, when implemented on devices with limited capabilities. As there exist automatic code generation tools and integrated development environments (IDE) for the DM6437 EVM as explained in [3], an initial working prototype has been obtained using Simulink and the Target Support Package. Such prototype has then been manually optimized and used as a test bench to implement and evaluate the SWAD-based tracker proposed in [4], as reported in section 5. Clearly, a reasonable follow-up 


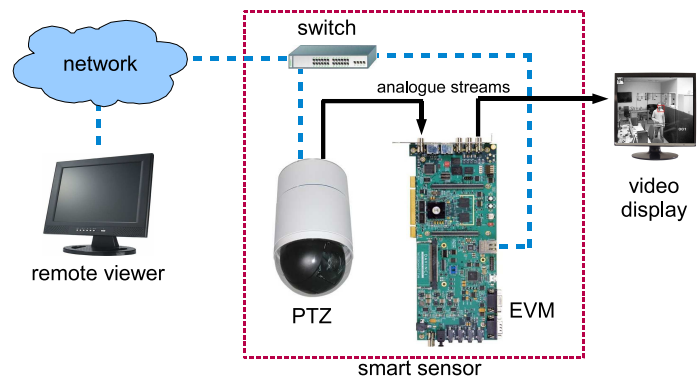

Fig. 1. Overview of the DSP embedded system, with the EVM and PTZ coupled together to form a smart surveillance sensor.

for the work presented in this paper would be to implement the same video analytics algorithm on an FPGA, for further performance evaluation.

The migration from a computer-based implementation of a tracking algorithm, like those described in [17], to a DSP embedded one is not straightforward. In such a context, the hardware available plays a fundamental part in the choice of the actual algorithm to implement. In this work, a PTZ camera is used, so that algorithms which rely strictly on the position of the target in the previous frames do not represent an ideal choice. For example, the Mean-Shift tracker (MS) [18] requires target overlapping in consecutive frames, within the so-called basin of attraction. However, this condition cannot be guaranteed with a moving camera.

On the other hand, DSP boards have limited resources, such as memory, bandwidth and word-length for number representation, so that not every tracking algorithm is suitable for an embedded implementation. In the proposed system, the hardware available is a DM6437 EVM equipped with a single core fixed-point DSP, that can perform integer operations on groups of 4-8 bytes in parallel. Therefore, on such DSP, algorithms working with floating point numbers would not take advantage of the architecture of the processor. Also in this case, the MS is not an ideal choice, as it heavily relies on floating point operations. Instead, two algorithms that can exploit such architecture are Normalized Cross-Correlation (NCC) and Sum of Absolute Differences (SAD) template matching [19]. As we will shown in section 5, the SAD algorithm gives better performance than NCC in terms of efficiency, since additions are performed much faster than multiplications. However, SAD fails in many cases when occlusion or noise pixels exist. In this paper the new Sum of Weighted Absolute Differences proposed in [4] is implemented, as it can deal with partial occlusions, offering better tracking performance than $\mathrm{SAD}$, as demonstrated in section 5 .

\section{DSP embedded system}

An illustration of the DSP embedded video analytics system described in this paper is shown in Fig. 1. The system hardware comprises two main components: 


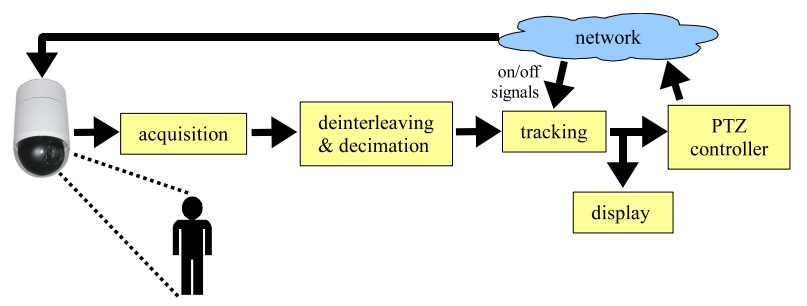

Fig. 2. Block diagram of the video analytics system.

a Spectrum Digital DM6437 Evaluation Module (EVM) equipped with a Texas Instruments TMS320DM6437 fixed-point DSP; and an ACTi IP Speed Dome CAM-6510, which is a pan-tilt-zoom (PTZ) camera with $360^{\circ}$ panning range, $180^{\circ}$ tilting range and a maximum angular speed of $400^{\circ}$ per second. A composite analogue video signal is also available as output from the PTZ and is fed into the EVM's video-in port. Both PTZ and EVM are connected to a local area network (LAN) through their Ethernet interfaces, so that they can communicate with each other via TCP/IP. For display purposes only, the EVM's video-out port is connected to a video display.

The system software is implemented in $\mathrm{C}$ and runs in real-time at more than 30 frames per second on the EVM. The PTZ camera hosts a proprietary web server and hence no software has been developed for it. Commands for the PTZ are encoded in HTTP requests to the camera web server. The video analytics algorithm on the EVM controls the PTZ by issuing such HTTP-based commands over the network. The EVM also runs a simple TCP server, so that remote on/off signals can be sent to the EVM, to activate the tracking algorithm.

When in stand-by mode, the system does not track any target and the PTZ can be moved freely. When the algorithm is activated, it starts tracking what is in the middle of the field of view at that exact moment. It is straightforward to integrate the proposed DSP embedded smart sensor with other event-based surveillance systems: for example an external smart system [20] can detect an event, compute the 3D position of the target, control the PTZ to point on the target, and then activate the SWAD-based tracking algorithm on the EVM, to follow the designated target.

\section{Embedded video analytics}

This section describes the video analytics implemented in our embedded system. A block diagram of it is shown in Fig. 2. Following the acquisition, deinterleaving and decimation processes, the output from the tracking block is the position of the target in the current frame. This information is used by the display block to highlight the target on the video display. The PTZ controller block uses the target position to decide whether to pan/tilt the PTZ camera. The tracking block also receives its own activation signals from the network. 

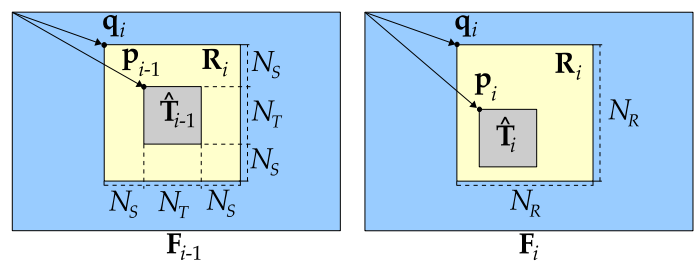

Fig. 3. Layout of the current frame $\mathbf{F}_{i}$ showing the best match $\hat{\mathbf{T}}_{i}$, the region of interest $\mathbf{R}_{i}$ and their positions within $\mathbf{F}_{i}$.

\subsection{Acquisition, deinterleaving and decimation}

The composite analogue video output from the PTZ is fed into the EVM's video-in port. This video stream is digitized into 8-bit interleaved $\mathrm{YCbCr}$ 4:2:2 frames of $576 \times 1440$ pixels, with $\mathrm{CbYCrY}$ packed format and video resolution of $576 \times 720$ pixels, by the video decoder present on the EVM board. A deinter-

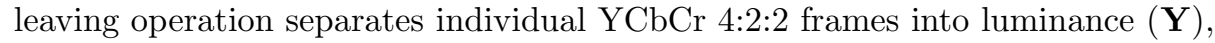
blue $\left(\mathbf{C}_{\mathbf{b}}\right)$ and red $\left(\mathbf{C}_{\mathbf{r}}\right)$ chrominance components, of which the tracking algorithm requires only $\mathbf{Y}$ to perform its task. The luminance component is then downsampled by a factor of 2 both vertically and horizontally, achieving a frame size of $288 \times 360$ pixels.

For speed optimization on the EVM, deinterleaving and decimation are performed at the same time, by simply extracting only the required samples from the 4:2:2 YCbCr frames. If $\mathbf{J}_{i}$ is the $i^{\text {th }}$ interleaved YCbCr 4:2:2 frame of $576 \times 1440$ pixels, the corresponding decimated luminance component $\mathbf{F}_{i}$ of $288 \times 360$ pixels is computed as:

$$
F_{i}(x, y)=J_{i}(2 x, 4 y+1): x \in[0, H-1], y \in[0, W-1]
$$

where $H=288$ and $W=360$ are height and width of $\mathbf{F}_{i}$.

\subsection{Adaptive template matching target tracking}

The tracking algorithm implemented on the DM6437 EVM is the adaptive template matching with minimization of a Sum of Weighted Absolute Differences (SWAD) described in [4]. The target model is represented by a template $\mathbf{T}_{i}$ of $N_{T} \times N_{T}$ pixels. As illustrated in Fig. 3, in the current frame $\mathbf{F}_{i}$ a region of interest $\mathbf{R}_{i}$ of $N_{R} \times N_{R}$ pixels, with $N_{R}=N_{T}+2 N_{S}$, is selected around the target position $\mathbf{p}_{i-1}$ in the previous frame $\mathbf{F}_{i-1}$. The best match $\hat{\mathbf{T}}_{i}$ in $\mathbf{F}_{i}$ for the target template $\mathbf{T}_{i}$ is found within the region of interest $\mathbf{R}_{i}$, by minimizing the SWAD coefficient $\psi(x, y)$ computed as:

$$
\psi(x, y)=\sum_{m=0}^{N_{T}-1} \sum_{n=0}^{N_{T}-1} K(m, n) \Delta(x, y, m, n)
$$


with pixel difference $\Delta(x, y, m, n)$ :

$$
\Delta(x, y, m, n)=\left|R_{i}(x+m, y+n)-T_{i}(m, n)\right|
$$

The kernel $\mathbf{K}$ in (2) is:

$$
K(x, y)=\left\lfloor 255 \cdot \frac{g(x, y)}{g(\lfloor\mu\rfloor,\lfloor\mu\rfloor)}\right\rfloor
$$

where $x, y \in\left[0, N_{T}-1\right]$ and $g(x, y)$ is a 2-dimensional Gaussian function with mean $\mu=\left(N_{T}-1\right) / 2$ and standard deviation $\sigma=N_{T} / 5$, defined as:

$$
g(x, y)=\exp \left(-\frac{(x-\mu)^{2}}{2 \sigma^{2}}-\frac{(y-\mu)^{2}}{2 \sigma^{2}}\right)
$$

Such kernel is used to assign high weights to central pixels and low weights to peripheral ones, as these pixels might belong to background or even occluding objects. In our DSP-based implementation, the values in $\mathbf{K}$ and the absolute differences in the SWAD metric are integers in the range $[0,255]$. The kernel values are computed offline once and stored in a look-up table. Using 8-bit integers allows us to optimally exploit the fixed-point architecture of the DM6437 DSP.

To take into account possible rescaling, rotation and target changes in general, the target template is updated using an infinite impulse response filter approach. Therefore the new target template $\mathbf{T}_{i+1}$ is computed as:

$$
\mathbf{T}_{i+1}=(1-\alpha) \mathbf{T}_{i}+\alpha \hat{\mathbf{T}}_{i}
$$

where $\alpha \in[0,1]$ is a blending factor. In our implementation it is $\alpha=0.5$. The position $\mathbf{p}_{i}$ in $\mathbf{F}_{i}$ of the best match $\mathbf{T}_{i}$ represent the position of the target in the current frame. This information is passed to the PTZ control block, to decide whether to pan/tilt the camera.

\subsection{PTZ controller}

The commands for the PTZ camera are hexadecimal sequences of 6 bytes included as parameters within standard HTTP requests, sent from the algorithm on the EVM to the web server running on the PTZ camera. The tracking algorithm establishes a TCP connection and sends the appropriate commands to the PTZ when the target approaches the frame boundaries. This situation is handled as follows. The current frame $\mathbf{F}_{i}$ is divided into horizontal and vertical regions, as shown in Fig. 4: horizontal-left (HL), horizontal-centre (HC) and horizontalright (HR); vertical-top (VT), vertical-centre (VC) and vertical-bottom (VB). For a given best match $\hat{\mathbf{T}}_{i}$ in $\mathbf{F}_{i}$, the PTZ pans to the left if $\hat{\mathbf{T}}_{i}$ overlaps the HL region, or to the right if $\hat{\mathbf{T}}_{i}$ overlaps HR; otherwise the camera does not move horizontally. Similarly, the PTZ tilts up if $\hat{\mathbf{T}}_{i}$ overlaps VT, or tilts down if $\hat{\mathbf{T}}_{i}$ overlaps $\mathrm{VB}$; otherwise the camera does not move vertically. This simple procedure allows the PTZ to follow the target, preventing it from going out of the field of view. 


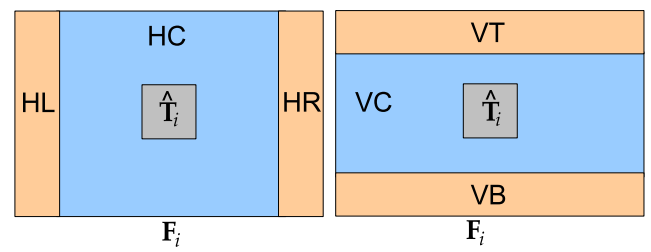

Fig. 4. Horizontal and vertical regions in the frame $\mathbf{F}_{i}$.
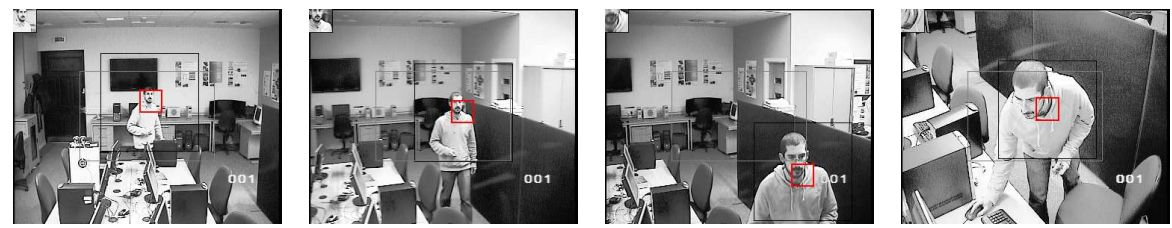

Fig. 5. Four images from the tracking algorithm running on the EVM.

\subsection{Video display}

The high resolution digital video output from the PTZ can be accessed over the network. For display purposes only, an analogue video signal is available from the EVM's video-out port. This signal contains the interleaved 4:2:2 $\mathrm{YCbCr}$ version $\mathbf{S}_{i}$ of the frame $\mathbf{F}_{i}$, with the values of the chrominance pixels set to 127 . The interleaved frame $\mathbf{S}_{i}$ is therefore a single plane matrix, with size equal to $H \times 2 W=288 \times 720$ pixels. The interleaving process is performed similarly to the deinterleaving process described in section 4.1. For every new incoming frame $\mathbf{F}_{i}$, only the pixels of $\mathbf{S}_{i}$ corresponding to the luminance component are modified and set equal to $\mathbf{F}_{i}$, while all the other pixels remains set to 127, as:

$$
S_{i}(x, y)=\left\{\begin{aligned}
F_{i}(x, m), & y=2 m+1 \\
127, & \text { otherwise }
\end{aligned}\right.
$$

where $x \in[0, H-1], y \in[0,2 W-1]$ and $m \in[0, W-1]$. Four frames from the video analytics algorithm running on the EVM are shown in Fig. 5. For display purpose, the best match $\hat{\mathbf{T}}_{i}$, the region of interest $\mathbf{R}_{i}$ and the vertical and horizontal regions described in section 4.3 are all highlighted on the display. Note that the current template $\mathbf{T}_{i}$ is shown on the top-left corner of each video frame.

\section{System performance evaluation}

In this section, a quantitative evaluation of the embedded system is given.

\subsection{Accuracy and precision}

A comparison of PC-based Matlab implementations of SWAD, SAD, NCC and MS has been carried out to assess the relative tracking capability of the SWAD- 
Table 1. Mean value $\mu_{\epsilon}$ and standard deviation $\sigma_{\epsilon}$ of error $\epsilon_{i}$ in pixels, and percentage $\lambda_{\epsilon}$ of frames with lowest error, for each tracker.

\begin{tabular}{|c|c|c|c|c|c|c|c|c|c|c|c|c|}
\hline & \multicolumn{3}{|c|}{$\mathrm{SAD}$} & \multicolumn{3}{|c|}{$\mathrm{NCC}$} & \multicolumn{3}{|c|}{ MS } & \multicolumn{3}{|c|}{ SWAD } \\
\hline & $\mu_{\epsilon}$ & $\sigma_{\epsilon}$ & $\lambda_{\epsilon}$ & $\mu_{\epsilon}$ & $\sigma_{\epsilon}$ & $\lambda_{\epsilon}$ & $\mu_{\epsilon}$ & $\sigma_{\epsilon}$ & $\lambda_{\epsilon}$ & $\mu_{\epsilon}$ & $\sigma_{\epsilon}$ & $\lambda_{\epsilon}$ \\
\hline$\overline{\text { Dudek }}$ & 4.25 & 2.54 & $7 \%$ & 4.29 & 2.36 & $24 \%$ & - & - & - & 3.67 & 2.13 & $34 \%$ \\
\hline PETS2006 & 30.99 & 22.56 & $0 \%$ & 34.13 & 24.21 & $0 \%$ & 14.19 & 4.44 & $7 \%$ & 10.91 & 4.14 & $93 \%$ \\
\hline PETS2007 & 6.67 & 6.22 & $15 \%$ & 9.93 & 9.24 & $11 \%$ & 7.34 & 1.35 & $7 \%$ & 3.89 & 1.50 & $53 \%$ \\
\hline PETS2009 & 4.27 & 3.25 & $37 \%$ & 27.38 & 47.80 & $7 \%$ & 81.95 & 97.72 & $0 \%$ & 2.26 & 1.42 & $48 \%$ \\
\hline
\end{tabular}

based adaptive template matching tracker, as shown in Fig. 6. Four publicly available test sequences have been used: Dudek face sequence [21], S1-T1-C/3 from PETS2006 dataset [22], S06_2/1 from PETS2007 dataset [23], and S3-multi12.43/8 from PETS2009 dataset [24]. As the Dudek sequence is in grey scale, the MS has not been applied to it. The ground truth for the Dudek sequence is already available, while the three PETS sequences have been manually labelled. For each frame $\mathbf{F}_{i}$, an error $\epsilon_{i}$ is computed in terms of Euclidean distance between the ground truth and the target position returned by the trackers. The accuracy in pixels of a tracker is therefore obtained as mean value $\mu_{\epsilon}$ of the error $\epsilon_{i}$ over a sequence, while the precision is computed as the standard deviation $\sigma_{\epsilon}$. With $\lambda_{\epsilon}$ we define the percentage of frames in a sequence in which a given tracker has the lowest error compared with the other trackers. In the left column of Fig. 6 the first frames with initial target positions for each sequence are shown, while in the right column graphs of the error $\epsilon_{i}$ are illustrated. Numerical values of $\mu_{\epsilon}$, $\sigma_{\epsilon}$ and $\lambda_{\epsilon}$ are reported in Table 1 . It can be seen that in general the error for SWAD is significantly lower, with better accuracy and precision, i.e. the lowest mean error $\mu_{\epsilon}$ and standard deviation $\sigma_{\epsilon}$. Moreover the SWAD-based tracker has also the lowest error for most of the frames, i.e. highest $\lambda_{\epsilon}$.

\subsection{Execution time}

After manual profiling and code optimization, the total running time of the DSP embedded SWAD-based adaptive template matching target tracking algorithm described in section 4 is of $15 \mathrm{~ms}$ per frame, giving a processing frame rate comfortably higher than the real-time requirement of $25-30 \mathrm{fps}$. The SWAD matching block in (2) and (3) with $N_{T}=32$ and $N_{S}=50$ takes 7 ms. Optimization for the SWAD matching is achieved by exploiting the C code "intrinsics", which are specific functions for the C6000 architecture of the DM6437 DSP [25]. Each C-level intrinsic function is mapped to a single assembly instruction and it executes additions, multiplications and absolute subtractions on groups of four 8-bit integers. For example, the intrinsic function _MEM4 reads 4 pixel values from memory; _SUBABS4 computes the absolute difference between two groups of 4 pixels; and _DOTPU4 computes the dot product between two vectors of 4 pixels. This approach reduces the number of operations for each row of pixels in the template $\mathbf{T}_{i}$ by a factor of 4 . A plain implementation of the SWAD matching without intrinsics takes $63 \mathrm{~ms}$, so being 9 times slower than optimized SWAD. 


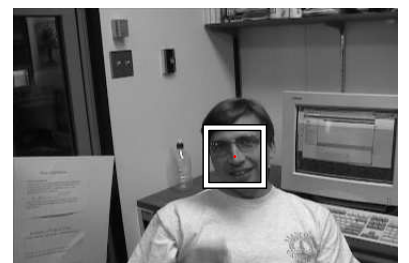

(a)

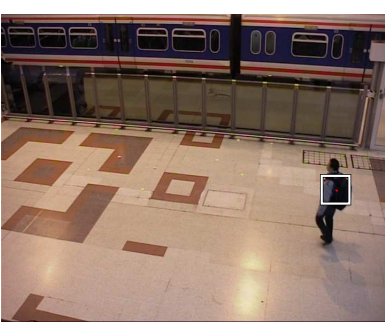

(c)

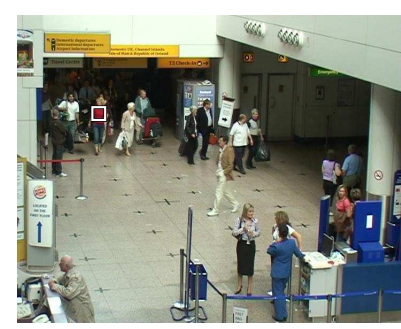

(e)

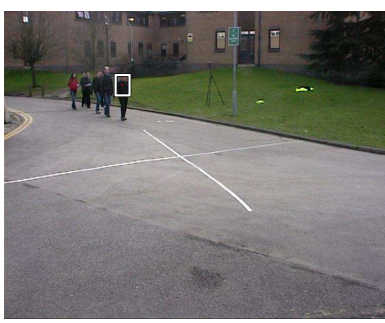

$(\mathrm{g})$

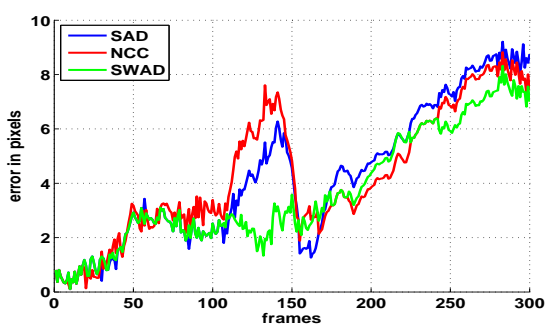

(b)

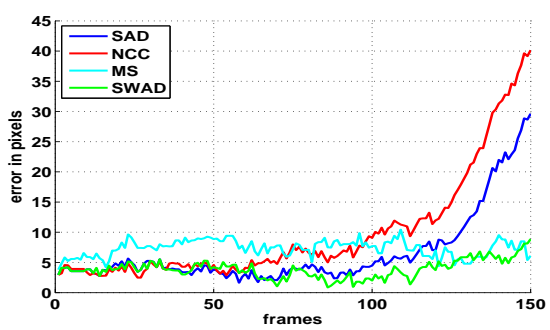

(d)

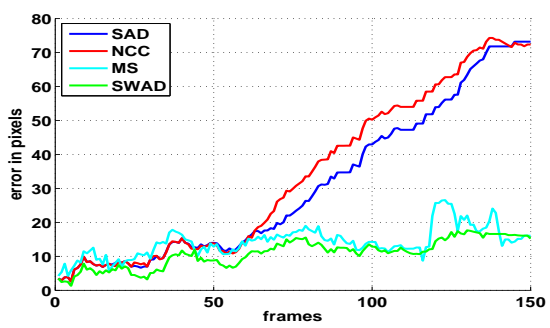

(f)

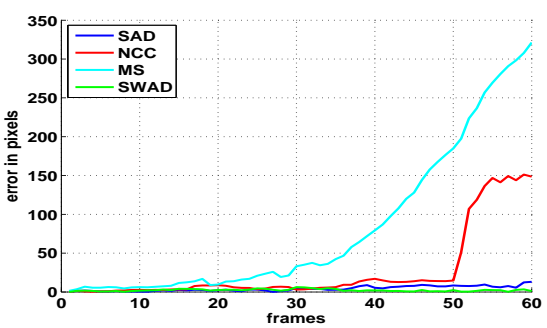

(h)

Fig. 6. Accuracy and precision test. (a-b) Dudek face sequence; (c-d) PETS2006; (ef) PETS2007; (g-h) PETS2009. (a-c-e-g) Initialization frames; (b-d-f-h) error distance from ground truth in pixels.

This demonstrates that the presented SWAD-based adaptive template matching algorithm exploits the fixed-point architecture of the DSP on the EVM. 
An optimized version of the SAD matching using intrinsic functions takes $5 \mathrm{~ms}$. Nonetheless, even though SWAD is slightly slower than SAD, its better tracking performance reported in section 5.1 entirely justifies its usage over conventional SAD. Concerning an implementation of NCC on the DM6437, it can be said that extra care must be taken to simulate floating point operations, as for example square root, in integer arithmetic. It takes about 9 ms just to compute the mean values of the template $\mathbf{T}_{i}$ and of each $N_{T} \times N_{T}$ subregion in the ROI $\mathbf{R}_{i}$. Thus it is clear that the execution of a complete implementation of NCC matching on the DM6437 DSP would definitely take longer than $7 \mathrm{~ms}$, and therefore NCC would be slower than SWAD.

\section{Conclusion}

In this paper we have presented a DSP embedded smart surveillance tracking sensor, using a PTZ camera to follow a target and always keep it central to the field of view. The system runs in real-time and it is implemented on the fixed-point single core DSP DM6437 Evaluation Module. The adaptive template matching tracking algorithm employs a robust Sum of Weighted Absolute Differences (SWAD) to maintain high accuracy under noise and partial occlusion when conventional SAD fails. The system can be used as a working framework to develop new real-time matching techniques for tracking and video analytics. The system can also be easily integrated with other surveillance systems, to create a collaborative network of smart surveillance sensors.

Currently the speed of the active camera is fixed and therefore the system might fail in case of (very) fast moving targets. It is planned to set the panning and tilting angular speed of the camera proportional to the speed of the target. In such a way, the system should be able to follow very fast moving targets. Also, a zooming capability is going to be incorporated in the smart sensor. Finally, a strategy for handling severe and complete occlusion will be also added.

\section{References}

1. Valera, A., Velastin, S.A.: Intelligent distributed surveillance systems: a review. IEE Proc. - Vision, Image and Signal Processing 152(2) (2005) 192-204

2. Dee, H., Velastin, S.A.: How close are we to solving the problem of automated visual surveillance? A review of real-world surveillance, scientific progress and evaluative mechanisms. Machine Vision and Applications 19(5-6) (2008) 329-343

3. Di Caterina, G., Hunter, I., Soraghan, J.: An embedded smart surveillance system for target tracking using a PTZ camera. In: European DSP Education and Research Conference. (2010) 165-169

4. Di Caterina, G., Soraghan, J.J.: Adaptive template matching algorithm based on swad for robust target tracking. IET Electronics Letters 48(5) (2012) 261-262

5. Hunter, I.: Overview of embedded DSP design. In: European Signal Processing Conference. (2009) 475-479

6. Kisacanin, B.: Examples of low-level computer vision on media processors. In: IEEE Conf. on Computer Vision and Pattern Recognition. (2005) 135-140 
7. Kisacanin, B., Nikolic, Z.: Algorithmic and software techniques for embedded vision on programmable processors. Signal Processing: Image Communication, Elsevier 25(5) (2010) 352-362

8. Wang, Y., Velipasalar, S., Casares, M.: Cooperative object tracking and composite event detection with wireless embedded smart cameras. IEEE Trans. on Image Processing 19(10) (2010) 2614-2633

9. Magno, M., Tombari, F., Brunelli, D., Di Stefano, L., Benini, L.: Multimodal abandoned/removed object detection for low power video surveillance systems. In: IEEE Int. Conf. on Advanced Video and Signal Based Surveillance. (2009) 188-193

10. Arth, C., Bischof, H.: Real-time object recognition using local features on a DSPbased embedded system. Journal of Real-Time Image Processing, Springer-Verlag 3(4) (2008) 233-253

11. Yang, C.S., Chen, R.H., Lee, C.Y., Lin, S.J.: PTZ camera based position tracking in IP surveillance system. In: Int. Conf. on Sensing Technology. (2008) 142-146

12. Kumar, P., Dick, A., Sheng, T.S.: Real time target tracking with pan tilt zoom camera. In: Digital Image Computing: Techniques and Applications. (2009) 492497

13. Chang, F., Zhang, G., Wang, X., Chen, Z.: PTZ camera target tracking in large complex scenes. In: World Congress on Intelligent Control and Automation. (2010) 2914-2918

14. Micheloni, C., Rinner, B., Foresti, G.L.: Video analysis in pan-tilt-zoom camera networks. IEEE Signal Processing Magazine 27(5) (2010) 78-90

15. McErlean, M.: An FPGA implementation of hierarchical motion estimation for embedded object tracking. In: IEEE Int. Symposium on Signal Processing and Information Technology. (2006) 242-247

16. McErlean, M.: Hierarchical motion estimation for embedded object tracking. In: IEEE Int. Symposium on Signal Processing and Information Technology. (2006) 797-802

17. Yilmaz, A., Javed, O., Shah, M.: Object tracking: a survey. ACM Computing Surveys 38(4) (2006) 1-45

18. Comaniciu, D., Ramesh, V., Meer, P.: Kernel-based object tracking. IEEE Trans. on Pattern Analysis and Machine Intelligence 25(5) (2003) 564-577

19. Tombari, F., Di Stefano, L., Mattoccia, S.: A robust measure for visual correspondence. In: Int. Conf. on Image Analysis and Processing. (2007) 376-381

20. Manap, N.A., Di Caterina, G., Ibrahim, M.M., Soraghan, J.J.: Co-operative surveillance cameras for high quality face acquisition in a real-time door monitoring system. In: European Workshop on Visual Information Processing. (2011) 99-104

21. Visual Tracking Benchmark: Dudek face sequence. http://www.cs.toronto.edu/vis/projects/adaptiveAppearance.html (2003)

22. PETS 2006: Benchmark Data. http://www.cvg.rdg.ac.uk/PETS2006/data.html (2006)

23. PETS 2007: Benchmark Data. http://www.cvg.rdg.ac.uk/PETS2007/data.html (2007)

24. PETS 2009: Benchmark Data. http://www.cvg.rdg.ac.uk/PETS2009/a.html (2009)

25. Texas Instruments: TMS320C6000 Programmer's Guide - SPRU198I. (March 2006) 